\title{
Iterative Decoding for MIMO Channels Via Modified Sphere Decoding
}

\author{
H. Vikalo, B. Hassibi, and T. Kailath
}

\begin{abstract}
In recent years, soft iterative decoding techniques have been shown to greatly improve the bit error rate performance of various communication systems. For multiantenna systems employing space-time codes, however, it is not clear what is the best way to obtain the soft information required of the iterative scheme with low complexity. In this paper, we propose a modification of the Fincke-Pohst (sphere decoding) algorithm to estimate the maximum a posteriori probability of the received symbol sequence. The new algorithm solves a nonlinear integer least squares problem and, over a wide range of rates and signal-to-noise ratios, has polynomial-time complexity. Performance of the algorithm, combined with convolutional, turbo, and low-density parity check codes, is demonstrated on several multiantenna channels. The results for systems that employ space-time modulation schemes seem to indicate that the best performing schemes are those that support the highest mutual information between the transmitted and received signals, rather than the best diversity gain.
\end{abstract}

Index Terms-Expected complexity, iterative decoding, low-density parity check (LDPC) codes, multiantenna systems, polynomial-time complexity, space-time codes, sphere decoding, turbo codes, wireless communications.

\section{INTRODUCTION}

$\mathbf{R}$ ECENTLY, the pursuit of high-speed wireless data services has generated a significant amount of activity in the communications research community. The physical limitations of the wireless medium present many challenges to the design of reliable communication systems. As shown in [1], multiantenna wireless communication systems are capable of providing data transmission at potentially very high rates. In multiantenna systems, space-time [2], [3] (along with traditional error-correcting) codes are often employed at the transmitter to induce diversity. Furthermore, to secure high reliability of the data transmission, special attention has to be payed to the receiver design. However, good decoding schemes may result in high complexity of the receiver.

A low-complexity detection scheme for multiantenna systems in a fading environment has been proposed in [4]. This detection scheme (so-called "nulling and cancelling"), depending on the adopted criterion, essentially performs zero-forcing or

Manuscript received February 21, 2003; revised July 21, 2003; accepted August 26,2003 . The editor coordinating the review of this paper and approving it for publication is M. Shafi. This work was supported in part by the National Science Foundation under Grant CCR-0133818, by the Office of Naval Research under Grant N00014-02-1-0578, and by the Lee Center for Advanced Networking, California Institute of Technology.

H. Vikalo and B. Hassibi are with the California Institute of Technology, Pasadena, CA 91125USA(e-mail: hvikalo@systems.caltech.edu; hassibi@systems.caltech.edu).

T. Kailath is with the Information Systems Laboratory, Stanford University, Stanford, CA 94309 USA.

Digital Object Identifier 10.1109/TWC.2004.837271 minimum-mean-square-error decision feedback equalization on block transmissions. In [5], a technique referred to as "sphere decoding" (based on the Fincke-Pohst algorithm [6]) was proposed for lattice code decoding and further adapted for space-time codes in [7]. The sphere decoder provides the maximum likelihood (ML) estimate of the transmitted signal sequence and so often significantly outperforms heuristic nulling and cancelling. Moreover, it was generally believed that sphere decoding requires much greater computational complexity than the cubic-time nulling and cancelling techniques. However, in [8], an analytic expression for the expected complexity of the sphere decoding has been obtained where it is shown that, over a wide range of rates and signal-to-noise ratios (SNRs), the expected complexity is polynomial-time (often subcubic). This implies that in many cases of interest ML performance can be obtained with complexity similar to nulling and cancelling.

Another area of intense research activity is that of soft iterative decoding. Such techniques have been reported to achieve impressive results for codes with long codeword length. Following the seminal paper by Berrou et al. [9], there have been many results on turbo decoding of concatenated codes, with performances approaching the Shannon limit on single-input single-output systems (see [10] and references therein). More recently, low-density parity check (LDPC) codes, long neglected since their introduction by Gallager [11], have also been resurrected (see, e.g., [12] and [13]).

Crucial to both turbo and LDPC decoding techniques is the use of the probabilistic ("soft") information about each bit in the transmitted sequence. For multiantenna systems employing space-time codes it is not clear what is the best way to obtain this soft -information with low complexity. As noted in [14], where turbo-coded modulation for multiantenna systems has been studied, if soft information is obtained by means of an exhaustive search, the computational complexity grows exponentially in the number of transmit antennas and in the size of the constellation. Hence, for high rate systems with a large number of antennas, the exhaustive search proves to be practically infeasible. Therefore, heuristics are often employed to obtain soft channel information [14]. [Also, see [15] and the references therein for a related work in the context of multiuser detection.] In [16] and [17], two variations of the sphere decoding algorithm have been proposed for estimating the soft information. In [17], sphere decoding has been employed to obtain a list of bit sequences that are "good" in a likelihood sense. This list is then used to generate soft information, which is subsequently updated by iterative channel decoder decisions. 
In this paper, we propose a multiple-input multiple-output (MIMO) detector, based on a modification of the original Fincke-Pohst algorithm, which efficiently obtains soft information for the transmitted bit sequence. This modified Fincke-Pohst algorithm essentially performs a maximum a posteriori (MAP) search and thus provides soft information for the channel decoder. The channel decoder's output is then fed back to the Fincke-Pohst MAP (FP-MAP) for the next iteration. [Note that the channel decoder may be iterative as well, as in the cases when the channel code is turbo or LDPC.] Our method differs from that of [17] in that the sphere decoder is modified (to allow for the introduction of soft information from the iterative decoder), that the detector performs MAP search, and that FP-MAP is repeated for each iteration. The fact that FP-MAP is repeated for each iteration does not necessarily mean that the technique of [17] is computationally more efficient. In particular, due to the feedback from the channel decoder, each consecutive iteration of FP-MAP results in a fewer number of points that are used to generate soft information, whereas the size of the list in [17] is kept fixed. Our very preliminary simulation results show that list sphere decoding and FP-MAP have comparable performance and complexity. Detailed quantitative analysis and comparison of the two techniques, and determining regimes where one is superior to the other, is certainly worth examining. However, it goes beyond the scope of this paper and will not be investigated here.

In addition to the schemes with traditional modulation techniques, we study the multiantenna systems employing space-time ( $\mathrm{S}-\mathrm{T}$ ) codes. S-T coding is a modulation technique that imposes spatial and temporal correlation onto a transmitted sequence of modulated symbols. It has been developed to fully exploit the spatial diversity provided by a wireless link. There has been a tremendous amount of the research activities in the field (see, e.g., [2] and [3]). In this paper, we focus on linear-dispersive (LD) codes of [19] and employ them in multiantenna systems where the transmitted data are encoded with powerful channel codes. We show that the LD codes allow for an efficient implementation of the FP-MAP algorithm, and illustrate the excellent performance of the proposed scheme via simulations. The $\mathrm{LD}$ codes are designed to optimize the mutual information between the transmitted and received signals. Maximizing the mutual information is a necessary condition to obtain the excellent performances promised by the powerful channel codes. We illustrate this by means of comparison with a space-time modulation scheme that does not optimize the above-mentioned mutual information-in particular, an orthogonal design [3].

This paper is organized as follows. The channel model and problem statement are in Section II. The Fincke-Pohst algorithm is described and the calculation of its expected complexity is outlined in Section III. In Section IV, we introduce the MAP modification of the Fincke-Pohst algorithm and discuss its complexity. In Section V, we present performance simulations of the FP-MAP algorithm in multiantenna systems both with and without space-time (LD) coding. The channel codes that we consider are convolutional, turbo, and LDPC codes. We conclude this paper in Section VI.

\section{SYSTEM MODEL}

We assume a discrete-time block-fading multiantenna channel model, where the channel is known to the receiver. This is a reasonable assumption for communication systems where the signaling rate is much faster than the pace at which the propagation environment changes, so that the channel may be learned, e.g., via transmitting known training sequences.

During any channel use the transmitted signal $\mathbf{s} \in \mathcal{C Z}^{M \times 1}$ and received signal $\mathbf{x} \in \mathcal{C}^{N \times 1}$ are related by

$$
\mathbf{x}=\sqrt{\frac{\rho}{M}} \mathbf{H s}+\mathbf{v}
$$

where $\mathbf{H} \in \mathcal{C}^{N \times M}$ is the known channel matrix and $\mathbf{v} \in$ $\mathcal{C}^{N \times 1}$ is the additive noise vector, both composed of independent, identically distributed complex-Gaussian entries $\mathcal{C N}(0$, 1). If we assume that the entries of $\mathbf{s}$ and $\mathbf{H}$ have unit variance, then $\rho$ is the expected received SNR. The channel is used multiple times to transmit a vector of data.

An iterative decoding scheme is shown in Fig. 1. The vector of information bits $\mathbf{b}$ is encoded with an error-correcting code to obtain the vector of coded bits $\mathbf{c}^{\prime}$, which is then interleaved to result in the vector $\mathbf{c}$. The vector $\mathbf{c}$ is modulated onto a quadrature amplitude modulation (QAM)-constellation. Assume that each constellation symbol represents $p_{m}$ modulated bits (e.g., for a $Q$-QAM constellation, $p_{m}=\log _{2} Q$ ). Then the modulation is performed by taking blocks of vector $\mathbf{c}$ of length $p_{m} M$ and mapping them (e.g., by means of a simple Gray mapping) into $M$-dimensional symbol vectors. The resulting symbols are transmitted across the channel as described by model (1). Therefore, a block of $p_{m} M$ coded bits (corresponding to a single symbol vector) is transmitted per each channel use. Let us denote these blocks of coded bits as $\mathbf{c}^{[1]}, \mathbf{c}^{[2]}, \ldots, \mathbf{c}^{\left[p_{c}\right]}$. Assume, for simplicity, that the total length of the vector $\mathrm{c}$ is $p_{c} p_{m} M$. Then the entire vector $\mathbf{c}$ can be blocked as

$$
\mathbf{c}=\left[\begin{array}{llll}
\mathbf{c}^{[1]} & \mathbf{c}^{[2]} & \cdots & \left.\mathbf{c}^{\left[p_{c}\right.}\right]
\end{array}\right]
$$

and transmitted in $p_{c}$ channel uses.

Consider that the $k$ th channel use (i.e., the block $\mathbf{c}^{[k]}$ has been modulated onto symbol vector $\mathbf{s}$ and transmitted across the channel). On the receiver side, the received vector $\mathbf{x}$ and a priori probabilities of the components of the symbol vector $\mathbf{s},\left\{p\left(\mathbf{s}_{1}\right), p\left(\mathbf{s}_{2}\right), \ldots, p\left(\mathbf{s}_{M}\right)\right\}$, are processed by an MIMO detector in order to obtain both the estimated bits in the current block $\mathbf{c}^{[k]}$ and the reliability information about those decisions. Let us denote bits in the block $\mathbf{c}^{[k]}$ by $c_{i}, i=1,2, \ldots, p_{m} M$. The reliabilities of the decisions for the coded bits $c_{i}$ can be expressed in the form of a log-likelihood ratio (LLR) as

$$
L_{1}\left(c_{i} \mid \mathbf{x}\right)=\log \frac{p\left[c_{i}=+1 \mid \mathbf{x}\right]}{p\left[c_{i}=-1 \mid \mathbf{x}\right]} .
$$

[Note: we will represent logical 0 with amplitude level -1 , and logical 1 with amplitude level +1 .] Let us denote the reliability information for the block $\mathbf{c}^{[k]}$ by

$$
\mathbf{L}_{1}^{[k]}=\left[L_{1}\left(c_{1} \mid \mathbf{x}\right) \quad L_{1}\left(c_{2} \mid \mathbf{x}\right) \quad \cdots \quad L_{1}\left(c_{p_{m} M} \mid \mathbf{x}\right)\right]
$$




\section{Transmitter:}

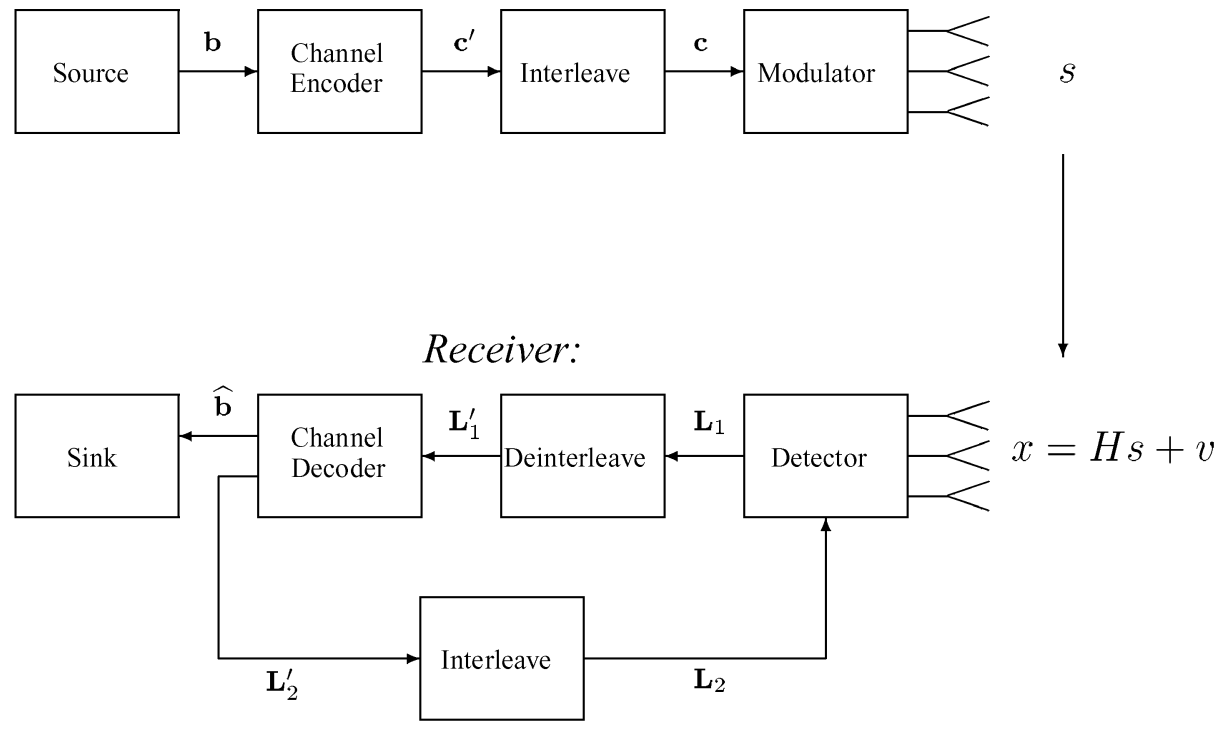

Fig. 1. System model.

and let $\mathbf{L}_{1}$ denote a vector of concatenated blocks of reliabilities

$$
\mathbf{L}_{1}=\left[\begin{array}{llll}
\mathbf{L}_{1}^{[1]} & \mathbf{L}_{1}^{[2]} & \cdots & \mathbf{L}_{1}^{\left[p_{c}\right]}
\end{array}\right]
$$

collected over all $p_{c}$ uses of the channel. Then $\mathbf{L}_{1}$ is a vector of LLRs corresponding to all the bits in the vector $\mathbf{c}$.

The vector $\mathbf{L}_{1}$ is deinterleaved to obtain vector $\mathbf{L}_{1}^{\prime}$, which is then used by a channel decoder to form the estimate of the information bit vector $\hat{\mathbf{b}}$, as well as to provide $\mathbf{L}_{2}^{\prime}$, the a posteriori reliability information for the coded bits vector $\mathbf{c}^{\prime}$. A posteriori reliability information for the vector $\mathbf{c}$ is obtained by deinterleaving $\mathbf{L}_{2}^{\prime}$ into $\mathbf{L}_{2}$. Let us denote the a posteriori reliability information for the block $\mathbf{c}^{[k]}$ by $\mathbf{L}_{2}^{[k]}$. Furthermore, assume that the bits $c_{i}, i=1,2, \ldots, p_{m} M$, in the block $\mathbf{c}^{[k]}$ are independent (which, for a long vector $\mathbf{c}$ and an efficient interleaver is a valid approximation). Then the a posteriori probabilities for the components of the symbol vector $\mathbf{s}$ (symbol vector corresponding to the block $\mathbf{c}^{[k]}$ ) can easily be found from $\mathbf{L}_{2}^{[k]}$ using the modulator mapping function. These probabilities $\left\{p\left(\mathbf{s}_{1}\right), p\left(\mathbf{s}_{2}\right), \ldots, p\left(\mathbf{s}_{M}\right)\right\}$ can now be used to run the MIMO detector algorithm [i.e., evaluate (3)] once again. Hence, the MIMO detector is an iterative one, and we use the described scheme for iterative joint detection and decoding in an MIMO system. [Note that for the first iteration of the MIMO detector, we assume that all symbols are equally likely.]

The structure of the channel decoder depends upon the choice of the error-correcting code. For a simple convolutional code, the channel decoder is a simple soft-in soft-out decoder, such as the Bahl, Cocke, Jelinek, and Raviv algorithm of [18]. When the channel code is a powerful turbo code, then the channel decoder is iterative itself [10]. If the channel code is an LDPC, the channel decoder employs message passing algorithms of [11].

The computational complexity of traditional algorithms for evaluating (3) can be prohibitive for applications in multiantenna systems. Since the sphere decoding algorithm of Fincke and Pohst can supply us with the ML estimate of $\mathbf{s}$ with reasonable complexity, one may speculate whether a modification can be devised to yield soft information with low complexity. To show that this can be done, and to describe how to efficiently approximate the LLRs in (3), it is instructive to review the original Fincke-Pohst algorithm [6].

\section{FInCKe-Pohst ALgORITHM}

We assume that the components of the transmitted symbol vector $\mathbf{s}$ in (1) are from a complex-valued QAM constellation. To state the ML detection as an integer least squares problem, we first find the real-valued equivalent of the (1). To this end, let $m=2 M, n=2 N$, and let $s, x$, and $v$ denote real vectors obtained from $\mathbf{s}, \mathbf{x}$, and $\mathbf{v}$, respectively, as

$$
\begin{aligned}
& s=\left[\begin{array}{ll}
\mathcal{R}(\mathbf{s})^{T} & \mathcal{I}(\mathbf{s})^{T}
\end{array}\right]^{T} \\
& x=\left[\begin{array}{ll}
\mathcal{R}(\mathbf{x})^{T} & \mathcal{I}(\mathbf{x})^{T}
\end{array}\right]^{T} \\
& v=\left[\begin{array}{ll}
\mathcal{R}(\mathbf{v})^{T} & \mathcal{I}(\mathbf{v})^{T}
\end{array}\right]^{T} .
\end{aligned}
$$

Furthermore, let $H \in \mathcal{R}^{n \times m}$ be given by

$$
H=\sqrt{\frac{\rho}{M}}\left[\begin{array}{cc}
\mathcal{R}(\mathbf{H}) & \mathcal{I}(\mathbf{H}) \\
-\mathcal{I}(\mathbf{H}) & \mathcal{R}(\mathbf{H})
\end{array}\right] .
$$

Then the real-valued equivalent of (1) is given by

$$
x=H s+v .
$$

The ML detector maximizes the likelihood that $x$ was received given that $s$ was sent

$$
\max _{s \in \mathcal{D}_{L}^{m}} p_{x \mid s}(x \mid s) .
$$

The search space $\mathcal{D}_{L}^{m}$ is a finite subset of the (shifted) $m$-dimensional integer lattice $\mathcal{Z}^{m}$, which reflects the fact that the unknown (complex) symbols $\mathbf{s}$ are from a QAM constellation. Therefore, $\mathcal{D}_{L}^{m}$ is an $L$-PAM constellation

$$
\mathcal{D}_{L}^{m}=\left\{-\frac{L-1}{2},-\frac{L-3}{2}, \ldots, \frac{L-3}{2}, \frac{L-1}{2}\right\}^{m}
$$

where $L$ is usually a power of two. 
Since $H$ is known and the noise is zero-mean unit-variance Gaussian, the conditional distribution of $x$ given $s$ is

$$
p_{x \mid s}(x \mid s)=\frac{1}{(2 \pi)^{m / 2}} e^{-\|x-H s\|^{2}} .
$$

Hence maximization (4) is equivalent to the optimization problem

$$
\min _{s \in \mathcal{D}_{L}^{m}}\|x-H s\|^{2}
$$

Problem (5) is referred to as an integer least squares problem and is known to be NP-hard. Geometrically, it corresponds to the search for the "closest" point in a skewed lattice $H \mathcal{D}_{L}^{m}$ to a given $n$-dimensional vector $x$.

The basic idea of the FP algorithm is that rather than search over the entire lattice, one should search only over lattice points in a hypersphere of radius $r$ around $x$. Then the closest lattice point inside the hypersphere is the solution to (5). To perform the search, however, one needs to 1) determine an appropriate radius $r$ and 2) find the lattice points inside the sphere.

The algorithm of Fincke and Pohst does not address the choice of $r$, but it does propose an efficient way of finding all the points inside the hypersphere. In particular, the algorithm constructs a tree, whose nodes at the $k$ th level correspond to the lattice points lying inside the sphere of radius $r$ and dimension $k$. To find the lattice points inside a sphere of radius $r$ and dimension $m$, the algorithm performs a depth-first tree search over all lattice points of radius $r$ and dimensions $k=1,2, \ldots, m$. The nodes in the tree that correspond to the points outside the sphere are pruned. This is illustrated in Fig. 2, for an $m=4$ dimensional lattice which has $L=4$ points in each dimension (i.e., $s \in \mathcal{D}_{4}^{4}$ ). [Remark: We need the tree-search interpretation for the discussion on the complexity of the algorithms; further details can be found in [8].]

The search radius $r$ can be chosen according to the statistical description of the noise. Note that $\|v\|^{2}=\|x-H s\|^{2}$ is a $\chi^{2}$ random variable with $n$ degrees of freedom. We choose the radius $r$ to be a linear function of the variance of $\|v\|^{2}$

$$
r^{2}=\alpha n
$$

where the coefficient $\alpha$ is chosen in such a way that with a high probability $p_{\mathrm{fp}}$ we find a lattice point inside a sphere

$$
\int_{0}^{\alpha n} \frac{\lambda^{n / 2-1}}{\Gamma(n / 2)} e^{-\lambda} d \lambda=p_{\mathrm{fp}}
$$

We find $\alpha$ in (6) by a simple table lookup.

\section{A. Computational Complexity of Fincke-Pohst Algorithm}

As noted above, the FP algorithm performs a search over all lattice points within a sphere of radius $r$ and dimensions $k=1,2, \ldots, m$. Hence the complexity of the algorithm is proportional to the number of lattice points visited. In general, the algorithm has worst case and average complexity that is exponential in the number of unknowns $m$ (see [8]). However, in communications applications, as is implied by (1), the vector $x$ is not arbitrary, but is a lattice point perturbed

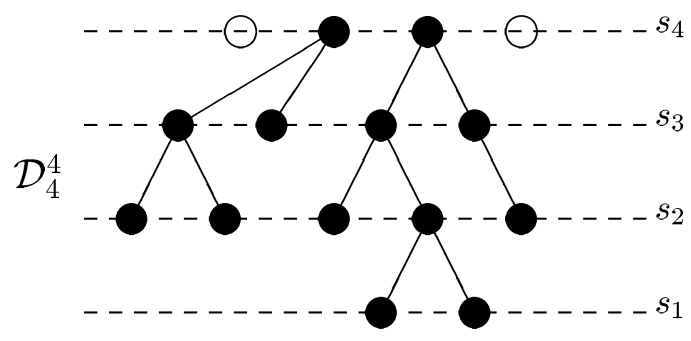

Fig. 2. Tree-pruning interpretation of sphere decoding.

by additive noise with known statistical properties. Hence, in this case, the expected complexity is a relevant figure of merit. The expected complexity of the FP algorithm is proportional to the expected number of lattice points that the algorithm visits. We need two key ingredients to calculate this expected number of lattice points (and, consecutively, the expected complexity).

1) A probability that an arbitrary lattice point $s_{a}$ belongs to a $k$-dimensional sphere of radius $r$ around the transmitted point $s_{t}$; it was shown in [8] that this probability is given by the following incomplete gamma function

$$
p_{s_{a}}=\gamma\left(\frac{\alpha n}{2\left(1+\frac{12 \rho}{m\left(L^{2}-1\right)}\left\|s_{a} s_{t}\right\|^{2}\right)}, \frac{n-m+k}{2}\right) .
$$

2) A technique for enumerating points $s_{a}$ in the lattice with respect to the transmitted point $s_{t}$; in [8], an efficient method for counting those lattice points that yield the same argument of the gamma function in (7), based on certain generating functions, is developed.

Using 1) and 2) above, one can find the number of lattice points visited by Fincke-Pohst algorithm and, therefore, the analytic expression for its expected complexity. The details can be found in [8], e.g., the complexity of the FP algorithm for a 2-pulse-amplitude-modulation (PAM) constellation is

$$
\begin{aligned}
C(m, \rho, \alpha)=\sum_{k=1}^{m}( & k+17) \sum_{l=0}^{k}\left(\begin{array}{l}
k \\
l
\end{array}\right) \gamma \\
& \times\left(\frac{\alpha n}{2\left(1+\frac{12 \rho l}{m\left(L^{2}-1\right)}\right)}, \frac{n-m+k}{2}\right) .
\end{aligned}
$$

For a 4-PAM constellation it is

$$
\begin{aligned}
C(m, \rho, \alpha)=\sum_{k=1}^{m}(2 & +17) \sum_{q} \frac{1}{2^{k}} \sum_{l=0}^{k}\left(\begin{array}{c}
k \\
l
\end{array}\right) g_{k l}(q) \gamma \\
& \times\left(\frac{\alpha n}{2\left(1+\frac{12 \rho q}{m\left(L^{2}-1\right)}\right)}, \frac{n-m+k}{2}\right)
\end{aligned}
$$

where $g_{k l}(q)$ is the coefficient of $x^{q}$ in the polynomial

$$
\left(1+x+x^{4}+x^{9}\right)^{l}\left(1+2 x+x^{4}\right)^{k-l} .
$$

Similar expressions can be obtained for 8-PAM, 16-PAM, etc., constellations.

For a wide range of $m, L$, and $\rho$, the sphere decoding algorithm has complexity comparable to cubic-time methods such 
as nulling and cancelling (cubic in $m$ ). As a general principle, for a fixed $m$, the complexity decreases by increasing the SNR $\rho$ or by decreasing $L$.

\section{MODIFIED FP ALGORITHM FOR MAP DETECTION}

The MAP detector maximizes the posterior probability $p_{s \mid x}(s \mid x)$

$$
\max _{s \in \mathcal{D}_{L}^{m}} p_{s \mid x}(s \mid x)
$$

Using Bayes' rule

$$
\begin{aligned}
\arg \max p_{s \mid x}(s \mid x) & =\arg \max \frac{p_{x \mid s}(x \mid s) p_{s}(s)}{p_{x}(x)} \\
& =\arg \max p_{x \mid s}(x \mid s) p_{s}(s)
\end{aligned}
$$

Further, by assuming that the symbols $s_{1}, s_{2}, \ldots, s_{m}$ are independent, we can write

$$
p_{s}(s)=\prod_{k=1}^{m} p\left(s_{k}\right)=e^{\sum_{k=1}^{m} \log p\left(s_{k}\right)} .
$$

Then, for a known channel in additive white Gaussian noise (AWGN), (10) is equivalent to the optimization problem

$$
\min _{s \in \mathcal{D}_{L}^{m}}\left[\|x-H s\|^{2}-\sum_{k=1}^{m} \log p\left(s_{k}\right)\right] .
$$

For an iterative decoding scheme, we also require soft information, i.e., the probability that each bit is decoded correctly. To this end, consider the LLR defined in (3) and, as in Section II, consider the $k$ th channel use (that is, the current symbol vector $s$ is obtained by modulating coded block $\mathbf{c}^{[k]}=\left[\begin{array}{llll}c_{1} & c_{2} & \cdots & c_{p_{m} M}\end{array}\right]$ onto an $L$-PAM constellation)

$$
\begin{aligned}
L_{1}\left(c_{i} \mid x\right) & =\log \frac{p\left[c_{i}=+1 \mid x\right]}{p\left[c_{i}=-1 \mid x\right]} \\
& =\log \frac{p\left[x, c_{i}=+1\right]}{p\left[x, c_{i}=-1\right]} \\
& =\log \frac{\sum_{\mathbf{c}^{[k]}: c_{i}=+1} p\left[x \mid \mathbf{c}^{[k]}\right] p\left[\mathbf{c}^{[k]}\right]}{\sum_{\mathbf{c}^{[k]}: c_{i}=-1} p\left[x \mid \mathbf{c}^{[k]}\right] p\left[\mathbf{c}^{[k]}\right]} .
\end{aligned}
$$

Assuming independent bits $c_{1}, c_{2}, \ldots, c_{p_{m} M},(12)$ becomes

$$
\begin{aligned}
L_{1}\left(c_{i} \mid x\right)= & \underbrace{\log \frac{p\left[c_{i}=+1\right]}{p\left[c_{i}=-1\right]}}_{L_{1 a}\left(c_{i}\right)} \\
& +\log \underbrace{\frac{\sum_{\mathbf{c}^{[k]}: c_{i}=+1} p\left[x \mid \mathbf{c}^{[k]}\right] \prod_{j, j \neq i} p\left[c_{j}\right]}{\sum_{\mathbf{c}^{[k]}: c_{i}=-1} p\left[x \mid \mathbf{c}^{[k]}\right] \prod_{j, j \neq i} p\left[c_{j}\right]}}_{L_{1 e}\left(c_{i}\right)}
\end{aligned}
$$

where $L_{1 a}\left(c_{i}\right)$ and $L_{1 e}\left(c_{i}\right)$ denote so-called a priori and extrinsic parts of the total soft information, respectively. [Note that, when used in an iterative decoding scheme, it is only $L_{1 e}\left(c_{i}\right)$ that is passed to the other decoding block(s) in the scheme.] Since the block $\mathbf{c}^{[k]}$ is uniquely mapped into the symbol vector $s$, it follows that for an AWGN channel

$$
\begin{aligned}
L_{1}\left(c_{i} \mid x\right) & =\log \frac{\sum_{s: c_{i}=+1} p[x \mid s] \prod_{j} p\left[s_{j}\right]}{\sum_{s: c_{i}=-1} p[x \mid s] \prod_{j} p\left[s_{j}\right]} \\
& =\log \frac{\sum_{s: c_{i}=+1} e^{-\|x-H s\|^{2}+\sum_{j} \log p\left[s_{j}\right]}}{\sum_{s: c_{i}=-1} e^{-\|x-H s\|^{2}+\sum_{j} \log p\left[s_{j}\right]}}
\end{aligned}
$$

Computing (13) over the entire signal space $\mathcal{D}_{L}^{m}$ is of prohibitive complexity. Instead, we constrain ourselves to those $s \in \mathcal{D}_{L}^{m}$ for which the argument in (11) is small. [Note that these are the signal vectors whose contribution to the numerator and denominator in (13) is significant.]

Applying the idea of the Fincke-Pohst algorithm, we search for the points $s$ that belong to the geometric body described by

$$
r^{2} \geq(s-\hat{s})^{*} R^{*} R(s-\hat{s})-\sum_{k=1}^{n} \log p\left(s_{k}\right)
$$

where $R$ is the lower triangular matrix obtained from the $\mathrm{QR}$ factorization of $H$. (Note that this is no longer a hypersphere.) The search radius $r$ in (14) can be chosen according to the statistical properties of the noise and the a priori distribution of $s$.

A necessary condition for $s_{m}$ to satisfy (14) readily follows:

$$
r_{m m}^{2}\left(s_{m}-\hat{s}_{m}\right)^{2}-\log p\left(s_{m}\right) \leq r^{2} .
$$

Moreover, for every $s_{m}$ satisfying (15), we define

$$
r_{m-1}^{2}=r^{2}-r_{m m}^{2}\left(s_{m}-\hat{s}_{m}\right)^{2}+\log p\left(s_{m}\right)
$$

and obtain a stronger necessary condition for (14) to hold

$$
r_{m-1, m-1}^{2}(s_{m-1}-\underbrace{\hat{s}_{m-1}+\frac{r_{m-1, m}}{r_{m-1, m-1}}\left(s_{m}-\hat{s}_{m}\right)}_{\hat{s}_{m-1 \mid m}})^{2}
$$

The procedure continues until all the components of vector $s$ are found. The FP-MAP algorithm can be summarized as follows.

Input: $R, x, \hat{s}, r, p_{s}(\mathbf{s})$.

1) Set $k=m, r_{m}^{\prime 2}=r^{2}-\|x\|^{2}+\|H \hat{s}\|^{2}, \hat{s}_{m \mid m+1}=\hat{s}_{m}$.

2) (Bounds for $s_{k}$ ) Set $z=\left(r_{k}^{\prime}\right) /\left(r_{k k}\right), U B\left(s_{k}\right)=\lfloor z+$ $\left.\hat{s}_{k \mid k+1}\right\rfloor, s_{k}=\left\lceil-z+\hat{s}_{k \mid k+1}\right\rceil-1$.

3) $\quad\left(\right.$ Increase $\left.s_{k}\right) s_{k}=s_{k}+1$. If $r_{k k}^{2}\left(s_{k}-\hat{s}_{k \mid k+1}\right)^{2}>r_{k}^{\prime 2}+$ $\log p\left(s_{k}\right)$ and $s_{k} \leq U B\left(s_{k}\right)$, go to 3$)$, else proceed. If $s_{k} \leq U B\left(s_{k}\right)$ go to 5), else to 4$)$. 
4) (Increase $k$ ) $k=k+1$; if $k=m+1$, terminate algorithm, else go to 3 ).

5) (Decrease $k$ ) If $k=1$ go to 6). Else $k=k-$ $1, \hat{s}_{k \mid k-1}=\hat{s}_{k}+\sum_{j=k+1}^{m}\left(r_{k j}\right) /\left(r_{k k}\right)\left(s_{j}-\hat{s}_{j}\right), r_{k}^{\prime 2}=$ $r_{k+1}^{\prime 2}-r_{k+1, k+1}^{2}\left(s_{k+1}-\hat{s}_{k+1 \mid k+2}\right)^{2}+\log p\left(s_{k+1}\right)$, and go to 2).

6) Solution found. Save $s$ and go to 3).

Assume that the search yields the set of points $\mathcal{S}=$ $\left\{s^{(1)}, s^{(2)}, \ldots, s^{\left(l_{s}\right)}\right\}$. The vector $s \in \mathcal{S}$ that minimizes (11) is the solution to the MAP detection problem (10). The soft information for each bit $c_{i}$ can be estimated from (13), by only summing the terms in the numerator and denominator such that $s \in \mathcal{S}$. Note that the logarithms in (13) can be efficiently computed using the standard log-MAP implementation [20].

\section{A. Computational Complexity of FP-MAP Algorithm}

The complexity of the FP-MAP algorithm can, in principle, be found following the outline of the calculation of complexity of the original Fincke-Pohst algorithm in Section III-A. However, the probability that an arbitrary point $s_{a}$ belongs to a $k$-dimensional sphere of radius $r$ around the transmitted point $s_{t}$ (which we need to compute the expected number of points the FP-MAP algorithm visits) now becomes

$$
p_{\mathbf{s}_{a}}=\gamma\left(\frac{\alpha n+\frac{12 \rho}{m\left(L^{2}-1\right)} \sum_{j=1}^{m} \log p\left(s_{j}\right)}{2\left(1+\frac{12 \rho}{m\left(L^{2}-1\right)}\left\|s_{a}-s_{t}\right\|^{2}\right)}, \frac{n-m+k}{2}\right) .
$$

The argument of this probability function is not as simple as the one in (7), and the computation of the expected number of points is much more difficult. First and foremost, (16) is a function of the a priori probabilities, which are generally not known in advance to iterations. Second, since each point $s_{a}$ in a lattice has a distinct a priori probability affiliated with it, argument of the probability function (16) will, in general, be different for each pair of points $\left(s_{t}, s_{a}\right)$. Thus an efficient enumeration that would help the complexity calculation cannot be done. Hence, to compute the expected number of points, one needs to consider all the possible pairs of points $\left(s_{t}, s_{a}\right)$ and the corresponding probabilities (16) which, as the size of the problem increases, clearly becomes rather cumbersome. However, we note that since $\log p\left(s_{j}\right) \leq 0, j=1, \ldots, m$, we have

$$
\alpha n+\frac{12 \rho}{m\left(L^{2}-1\right)} \sum_{j=1}^{m} \log p\left(s_{j}\right) \leq \alpha n .
$$

Hence from (7) and (16) it follows that, for the same choice of radius $r$

$$
p_{s_{a}}^{\mathrm{FP}-\mathrm{MAP}} \leq p_{\boldsymbol{s}_{a}}^{\mathrm{FP}}
$$

and we conclude that, for the same choice of $r$, the expected number of points that the FP-MAP algorithm visits is upper bounded by the expected number of points visited by the original sphere decoding algorithm. Thus, the expected complexity of the FP-MAP is roughly upper bounded by the expected complexity of the sphere decoding, for the same choice of $r$. ["Roughly" upper bounded because since the a priori probabilities enter the algorithm, there are a few (two, to be exact) additional operations per each visited point; this is accounted for by changing $(2 k+17)$ to $(2 k+19)$ in (8) and (9).]

Therefore, the results of [8] suggest that the expected complexity of the FP-MAP algorithm is polynomial in $m$ over a wide range of rates and SNRs. Generally, we chose the search parameter $r$ so that there are sufficiently many points to make a good approximation of (13).

\section{Simulation Results}

In this section, we study performances of several multiantenna systems employing the FP-MAP algorithm for iterative decoding.

Example 1 [Convolutional Code]: We consider the multiantenna system with $M=4$ transmit and $N=4$ receive antennas. An information bit sequence with 9216 information bits is encoded by a rate $R=1 / 2$ convolutional code with memory length 2 and generating polynomials $G_{1}(D)=1+D^{2}$ (feedforward) and $G_{2}(D)=1+D+D^{2}$ (feedback). The coded sequence is modulated by means of simple Gray mapping onto a 16-QAM modulation scheme. On the receiver side, the FP-MAP algorithm is used for soft detection.

Fig. 3 shows the bit error rate (BER) performance of the system compared with the performance of the system employing maximum likelihood (hard) detection. The dashed line in Fig. 3 denotes the SNR corresponding to the ergodic capacity of the channel.

Fig. 4 compares the performance of the FP-MAP with that of the soft nulling and cancelling (N/C) algorithm employed in the same system. For each entry in a transmitted symbol vector, the soft N/C algorithm cancels the previously decoded symbols and obtains the soft information using the distribution of the noise. [The soft N/C algorithm is similar to the soft minimum mean squared error equalizer of [21]. Also, see [15] for an application in multiuser context.] Prior to the soft N/C, symbols are optimally ordered. The complexity of the soft N/C algorithm is roughly cubic (due to the required QR-factorization of the channel matrix). The FP-MAP even with a single iteration outperforms four iterations of the soft N/C by $2 \mathrm{~dB}$ at BER of $10^{-4}$.

Example 2 [Turbo and LDPC Codes]: We study the multiantenna system of Example $1(M=N=4)$, where the transmitted data are encoded with powerful turbo and LDPC codes.

First consider the case where the 9216-bits-long information sequence is encoded with a rate $R=1 / 2$ parallel concatenated turbo code. The constituent convolutional codes of the turbo code are the same as the convolutional code described in Example 1. The modulation scheme is 16-QAM. The FP-MAP algorithm is used to obtain soft information, which is passed onto the turbo decoder. For each iteration of the FP-MAP, the turbo decoder performs eight iterations of its own. Fig. 5 shows the BER performance of the system.

Fig. 6 shows the BER performance of the same system $(M=$ $N=4$ ), but now the data are encoded with a rate $R=8 / 9$ LDPC code of length 1088 and column weight 4 . Furthermore, 4-QAM modulation scheme is employed. When the LDPC decoder receives soft information from FP-MAP, it performs eight iterations before passing what it infers about the coded bits back to the FP-MAP detector. 


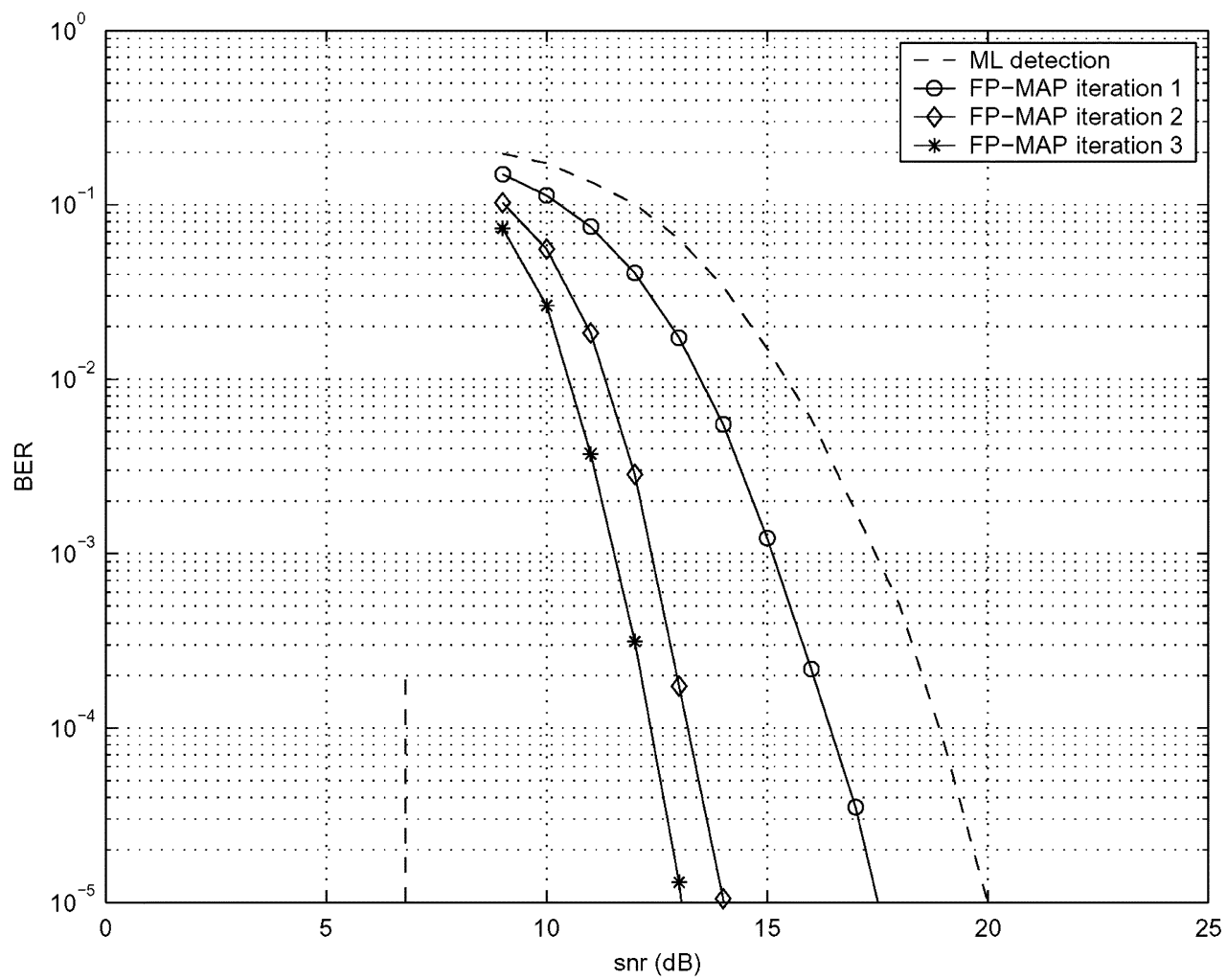

Fig. 3. BER performance of $M=N=4$ system employing rate 1/2, 9216-bits-long convolutional code, 16-QAM, FP-MAP.

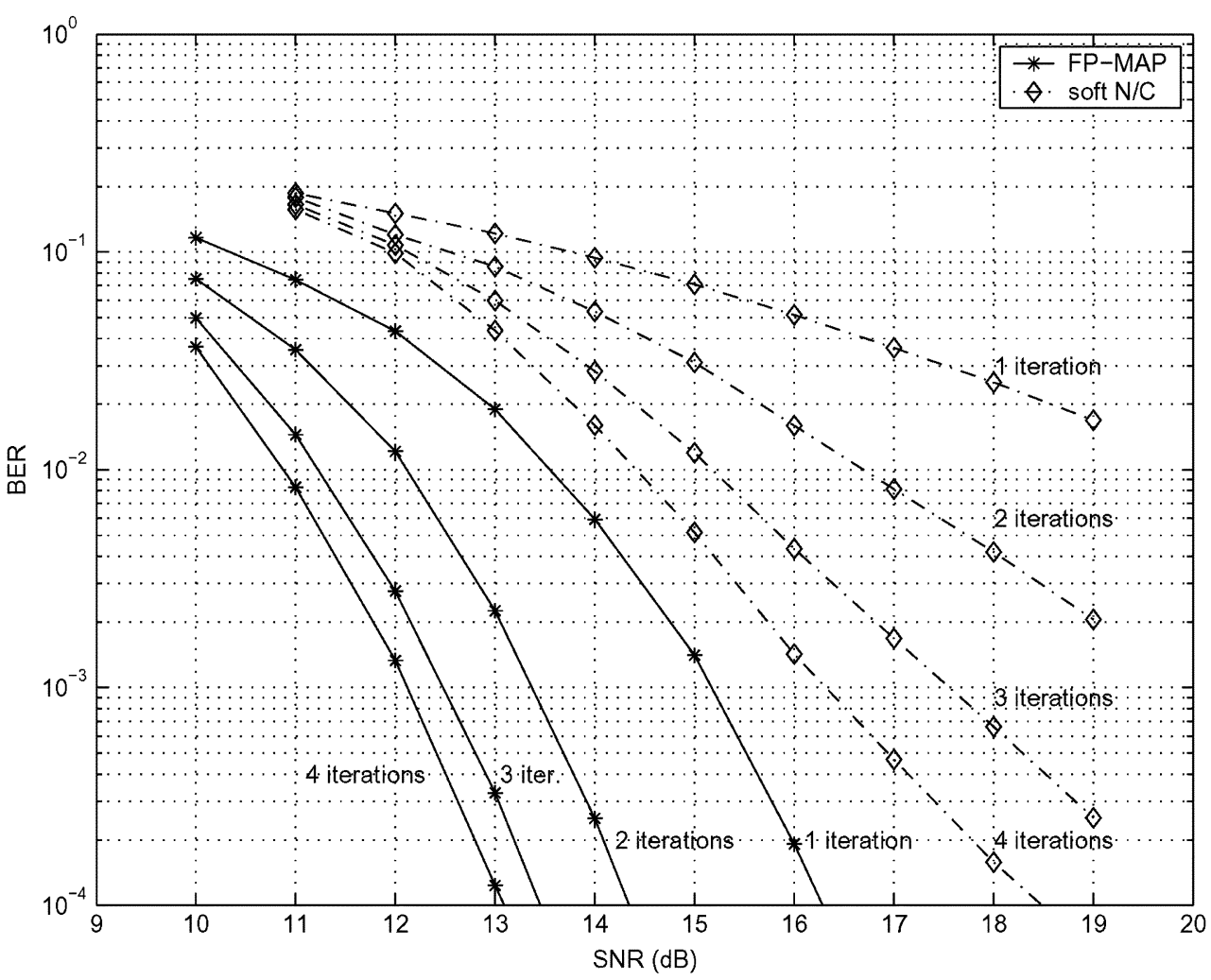

Fig. 4. Comparison of BER performances for FP-MAP and soft N/C employed on $M=N=4$ system with rate 1/2, 1000-bits-long convolutional code, 16-QAM.

Following the convention, the dashed vertical lines in Figs. 5 and 6 denote the capacity limits, i.e., they denote the smallest SNR required for reliable transmission at the given data rate of the system. This means that the error probability of the best code transmitting at smaller SNR than the one denoted by the dashed lines approaches one as the code length approaches infinity. The 


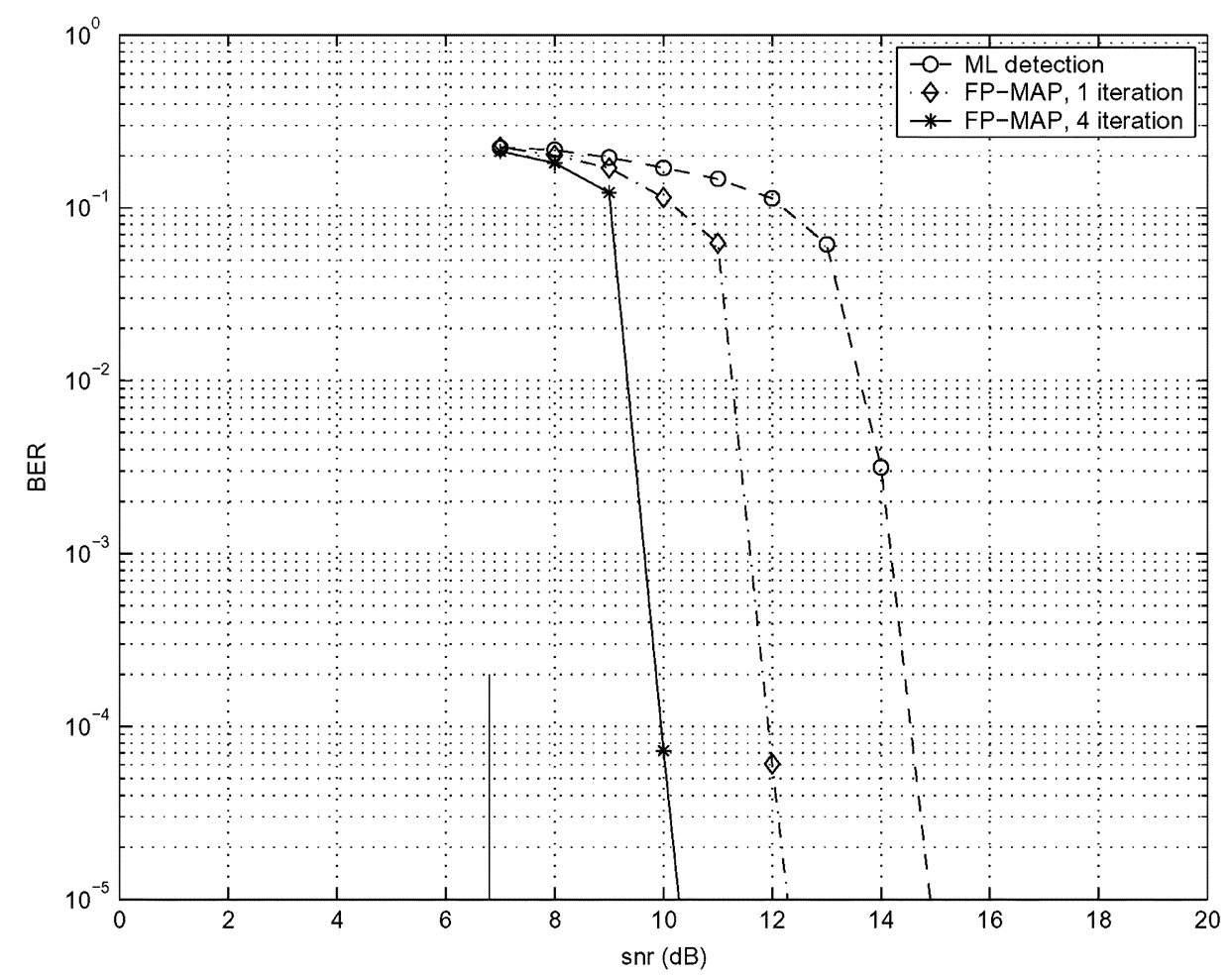

Fig. 5. BER performance of $M=N=4$ system employing rate $1 / 2$ turbo code, 16-QAM, FP-MAP.

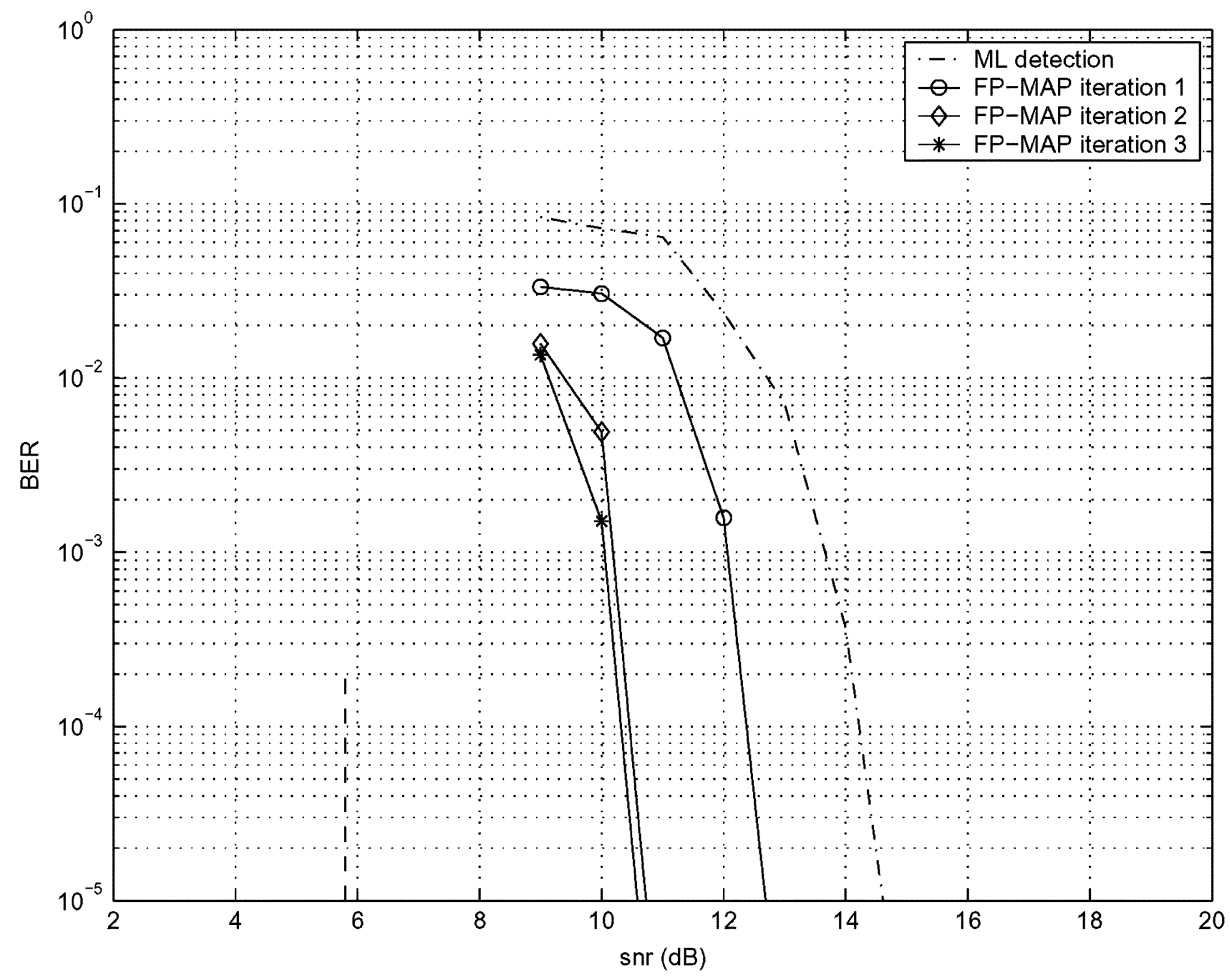

Fig. 6. BER performance of $M=N=4$ system employing rate 8/9 LDPC code, 4-QAM, FP-MAP.

turbo coded scheme in Fig. 5 gets $3.3 \mathrm{~dB}$ close to capacity. At BER of $10^{-5}$, it outperforms the convolutional code with performance in Fig. 3 by approximately $3 \mathrm{~dB}$. The rate of the system is 8 bits per channel use. The LDPC code, on the other hand, is about $4.5 \mathrm{~dB}$ away from capacity of the system in which it is employed; the data rate in this system is 7.1 bits per channel use.
Although the LDPC code is outperformed by the turbo code, it proves to be an interesting alternative, especially in light of the complexity exponents $\log _{m} \mathcal{C}(m, L, \rho)$ shown in Fig. 7. At $\mathrm{SNR} \approx 10 \mathrm{~dB}$, both schemes have BER $\approx 10^{-5}$. As indicated in Fig. 7, for such SNR, the complexity of the detection in the system employing the (high rate) LDPC code and 4-QAM mod- 

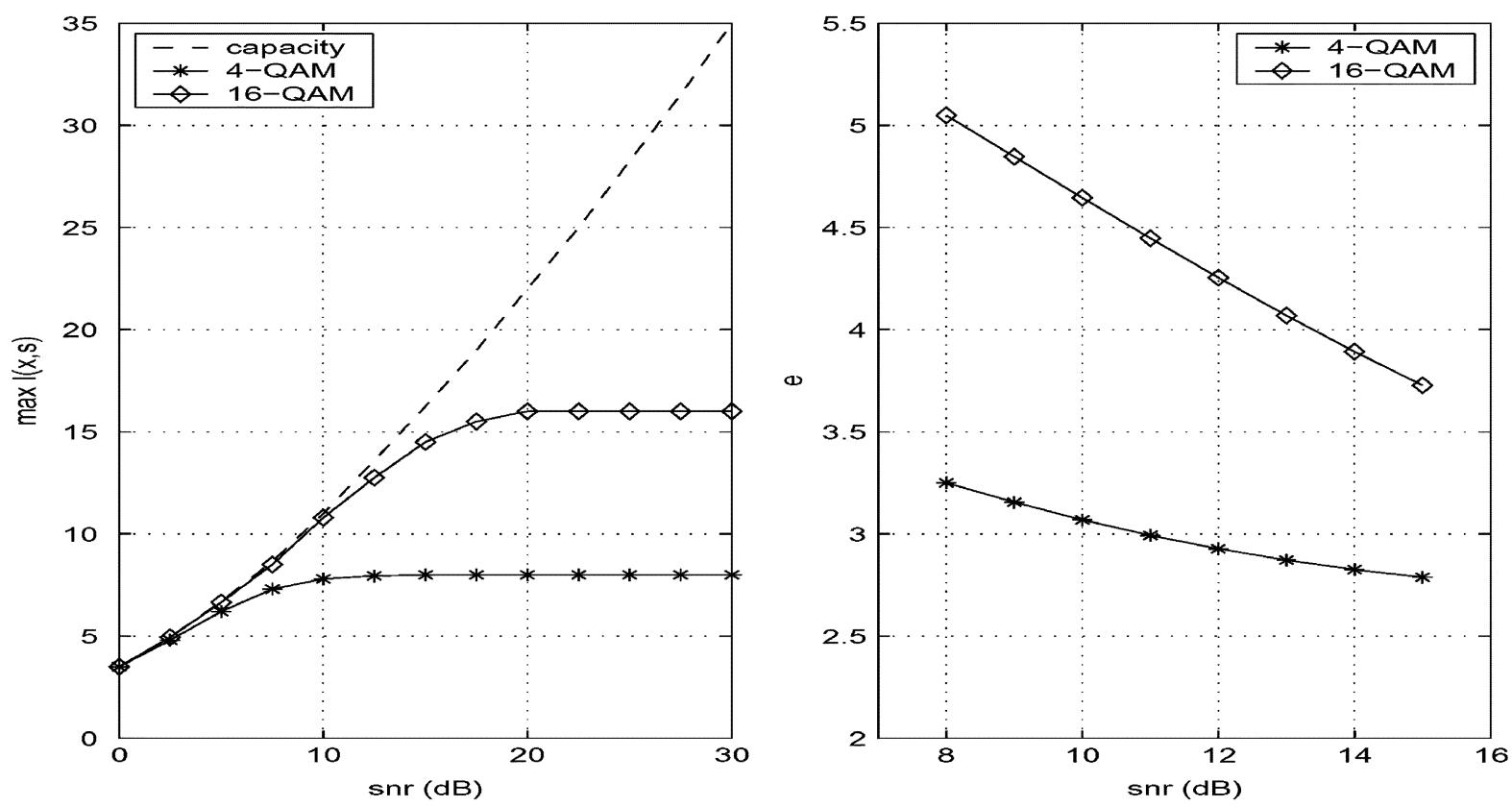

Fig. 7. Maximum mutual information and complexity exponents for 4-QAM and 16-QAM constellations, $M=N=4$ system.

ulation is approximately cubic in $m$, while the complexity in the system with the (1/2 rate) turbo code and 16-QAM modulation is significantly higher. So, although the mutual information plots in Fig. 7 imply that the system with 16-QAM modulation scheme is more efficient, the system that employs 4-QAM modulation is much more favorable from the complexity point of view.

Example 3 [Systems Employing Both Channel and Space-Time Codes]: Here we consider performance of the LDPC codes in a multiantenna system with linear dispersive (LD) space-time modulation scheme of [19]. A linear-dispersion code imposes correlation onto a symbol sequence by transmitting (over $T$ channel uses) columns of the matrix $S$ defined as

$$
S=\sum_{q=1}^{Q}\left(s_{q} C_{q}+s_{q}^{*} D_{q}\right)
$$

where $C_{q} \in \mathcal{C}^{T \times M}$ and $D_{q} \in \mathcal{C}^{T \times M}$ are fixed matrices, and $s_{q}, q=1, \ldots, Q$ are complex scalars. The particular choice of scalars $s_{1}, \ldots, s_{Q}$, typically coming from a QAM constellation, determines a specific codeword from the code that is determined by the set of matrices $\left\{C_{q}, D_{q}\right\}$. The rate of the $\mathrm{LD}$ code is $R_{S T}=(Q / T) \log _{2} r$.

Assume that $T=M=N$. By collecting vectors $\mathbf{x}_{1}, \ldots, \mathbf{x}_{N}$ received over $N$ uses of channel $\mathbf{H}$ into matrix $X$, and collecting corresponding noise vectors $\mathbf{v}_{1}, \ldots, \mathbf{v}_{N}$ into matrix $V$, the input/output relation of the multiantenna system can be expressed as

$$
X=\sqrt{\frac{\rho}{M}} S \mathbf{H}+V .
$$

In [19], it was shown that (17) can be written as

$$
x=\mathcal{H} s+v
$$

where $x=\left[\mathcal{R}\left(\mathbf{x}_{1}\right) \quad \mathcal{I}\left(\mathbf{x}_{1}\right) \cdots \mathcal{R}\left(\mathbf{x}_{N}\right) \quad \mathcal{I}\left(\mathbf{x}_{N}\right)\right]^{*}, s=$ $\left[\begin{array}{llll}\mathcal{R}\left(s_{1}\right) & \mathcal{I}\left(s_{1}\right) \cdots \mathcal{R}\left(s_{Q}\right) & \mathcal{I}\left(s_{Q}\right)\end{array}\right]^{*}, \quad$ and $\quad v \quad=$ $\left[\begin{array}{llll}\mathcal{R}\left(\mathbf{v}_{1}\right) & \mathcal{I}\left(\mathbf{v}_{1}\right) \cdots \mathcal{R}\left(\mathbf{v}_{N}\right) & \mathcal{I}\left(\mathbf{v}_{N}\right)\end{array}\right]^{*} . \quad$ Furthermore, the $2 N T \times 2 Q$ real valued equivalent channel matrix $\mathcal{H}$ is given by

$$
\mathcal{H}=\sqrt{\frac{\rho}{M}} H\left[\begin{array}{ccccc}
\mathcal{A}_{1} \underline{h}_{1} & \mathcal{B}_{1} \underline{h}_{1} & \cdots & \mathcal{A}_{Q} \underline{h}_{1} & \mathcal{B}_{Q} \underline{h}_{1} \\
\vdots & \vdots & \ddots & \vdots & \vdots \\
\mathcal{A}_{1} \underline{h}_{N} & \mathcal{B}_{1} \underline{h}_{N} & \cdots & \mathcal{A}_{Q} \underline{h}_{N} & \mathcal{B}_{Q} \underline{h}_{N}
\end{array}\right]
$$

where

$$
\begin{aligned}
\mathcal{A}_{q} & =\left[\begin{array}{ll}
\mathcal{R}\left(A_{q}\right) & -\mathcal{I}\left(A_{q}\right) \\
\mathcal{I}\left(A_{q}\right) & \mathcal{R}\left(A_{q}\right)
\end{array}\right] \\
\mathcal{B}_{q} & =\left[\begin{array}{ll}
-\mathcal{I}\left(B_{q}\right) & -\mathcal{R}\left(B_{q}\right) \\
\mathcal{R}\left(B_{q}\right) & -\mathcal{I}\left(B_{q}\right)
\end{array}\right] \\
\underline{h}_{n} & =\left[\begin{array}{ll}
\mathcal{R}\left(h_{n}\right) \\
\mathcal{I}\left(h_{n}\right)
\end{array}\right]
\end{aligned}
$$

where $A_{q}=C_{q}+D_{q}, B_{q}=C_{q}-D_{q}$, and where the vector $h_{n}, n=1, \ldots, N$, denotes the $n$th column of the MIMO channel matrix $\mathbf{H}$.

Note that entries of the vector $s$ in (18) come from an $L$-PAM constellation. Therefore, one can pose the MAP detection problem as

$$
\min _{s}\|x-\mathcal{H} s\|^{2}+\sum \log p\left(s_{i}\right)
$$

where $p\left(s_{i}\right)$ is the a priori probability of the $i$ th component of the vector $s$. Equation (19) allows for an efficient implementation of the FP-MAP algorithm.

We consider a multiantenna system where the data are encoded by the $R=8 / 9$-rate LDPC code of length 1088 (the same LDPC code is used in Example 2). Fig. 8 shows the performance of the $2 \times 2$ system employing the 4 -QAM modulation scheme 


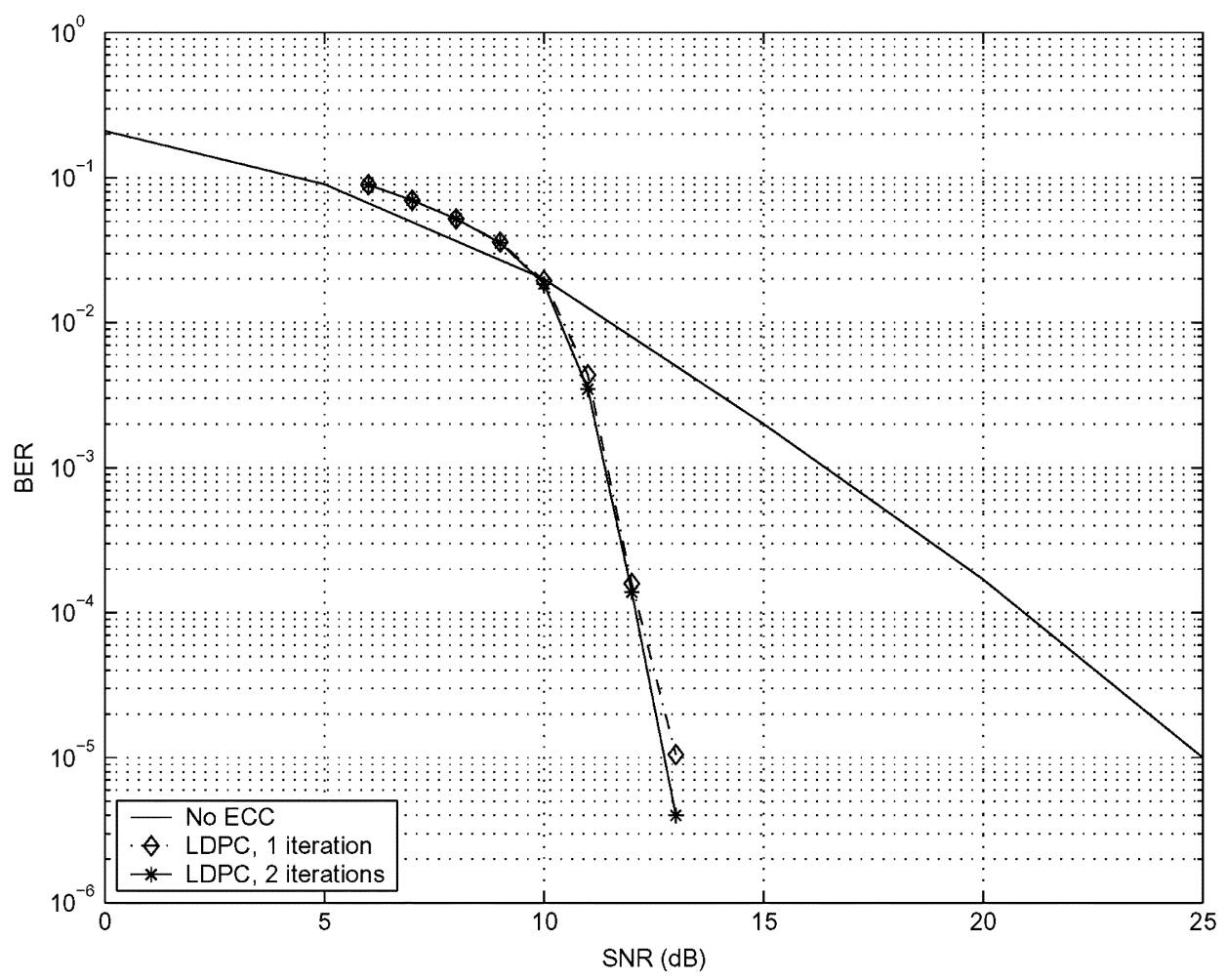

Fig. 8. BER performance of $M=N=2$ system employing $R=4 \mathrm{LD}$ code and rate $8 / 9$ LDPC code, 4-QAM modulation, FP-MAP.

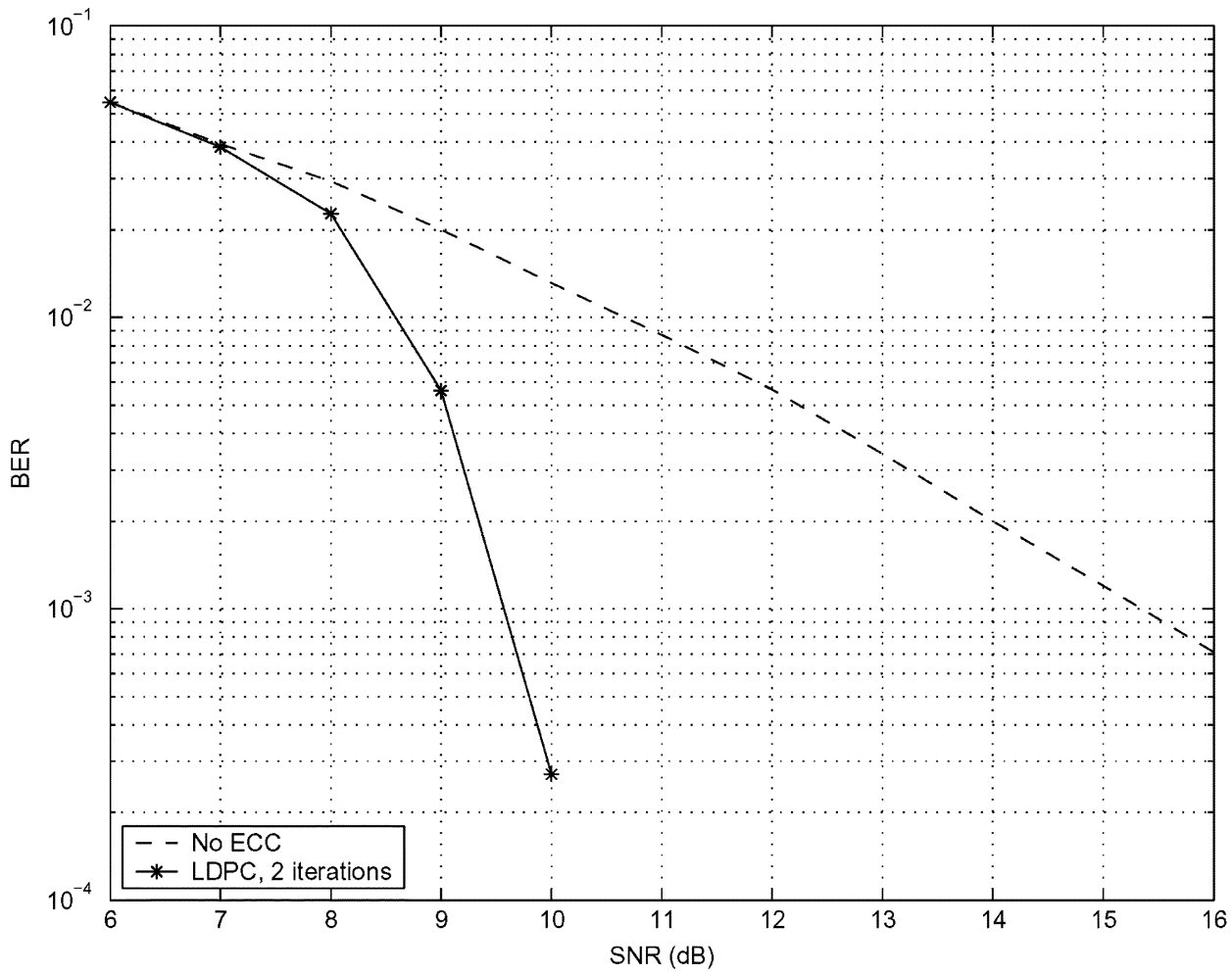

Fig. 9. BER performance of $M=3, N=1$ system employing $R=2$ LD code and rate 8/9 LDPC code, FP-MAP.

and a rate $R_{\mathrm{ST}}=4 \mathrm{LD}$ code (see [19, Section 4] for the construction of this $L D$ code). The significant coding gain due to use of the LDPC code is evident.Even without S-T coding, LDPC code would yield good performance, as illustrated in Example 2. However, S-T coding is necessary in systems with fewer re- ceive than transmit antennas, to provide as many equations as there are unknowns and ensure feasibility of decoding (when $M>N$, the Fincke-Pohst algorithm is exponential in $M-N$ ). Performance of such a system, with $M=3$ and $N=1$, employing an LD code is shown in Fig. 9. 


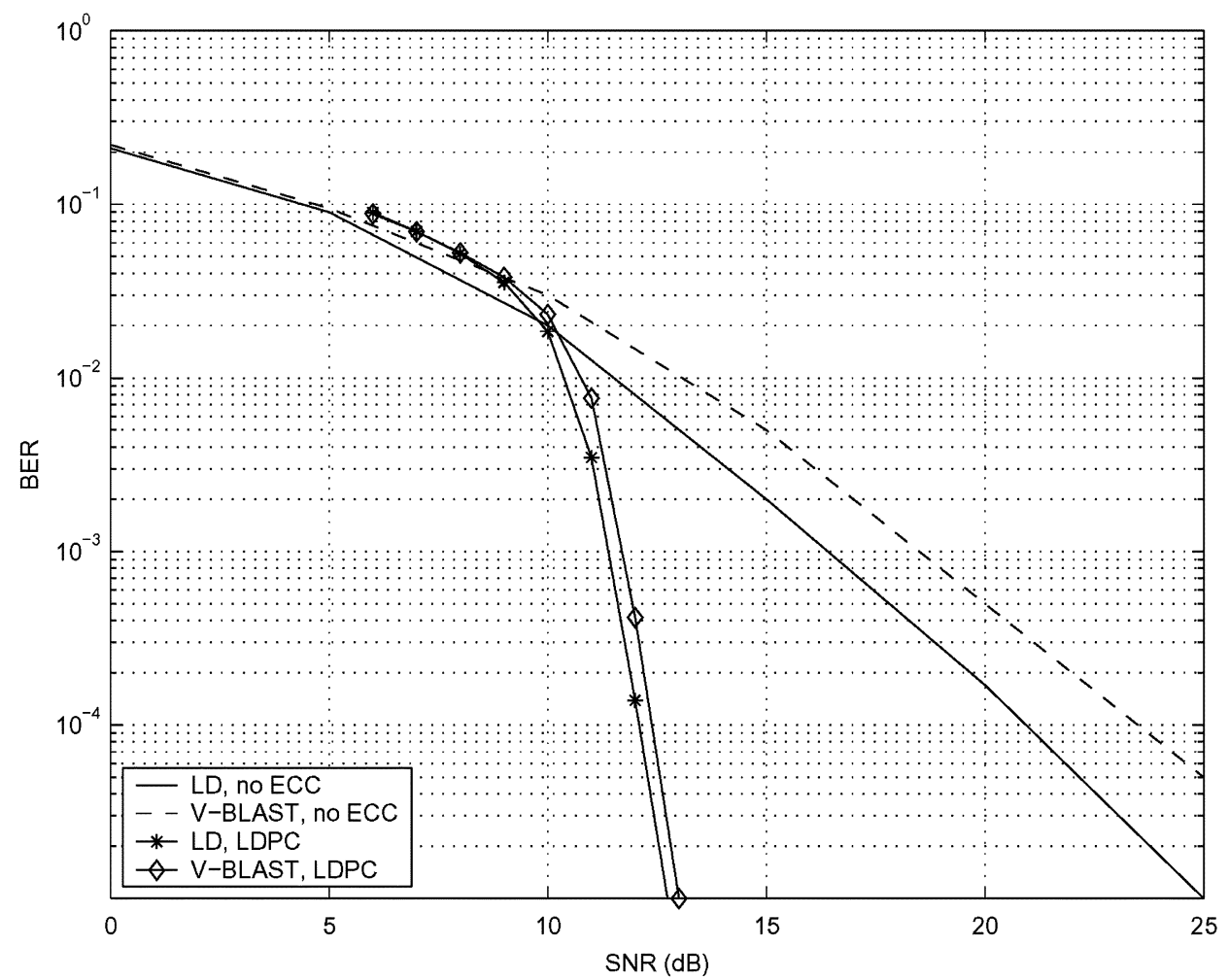

Fig. 10. Comparison of BER performances of LD code vs. V-BLAST (rate $R=4$ ) employed on $M=N=2$ system, using rate 8/9 LDPC code, FP-MAP.

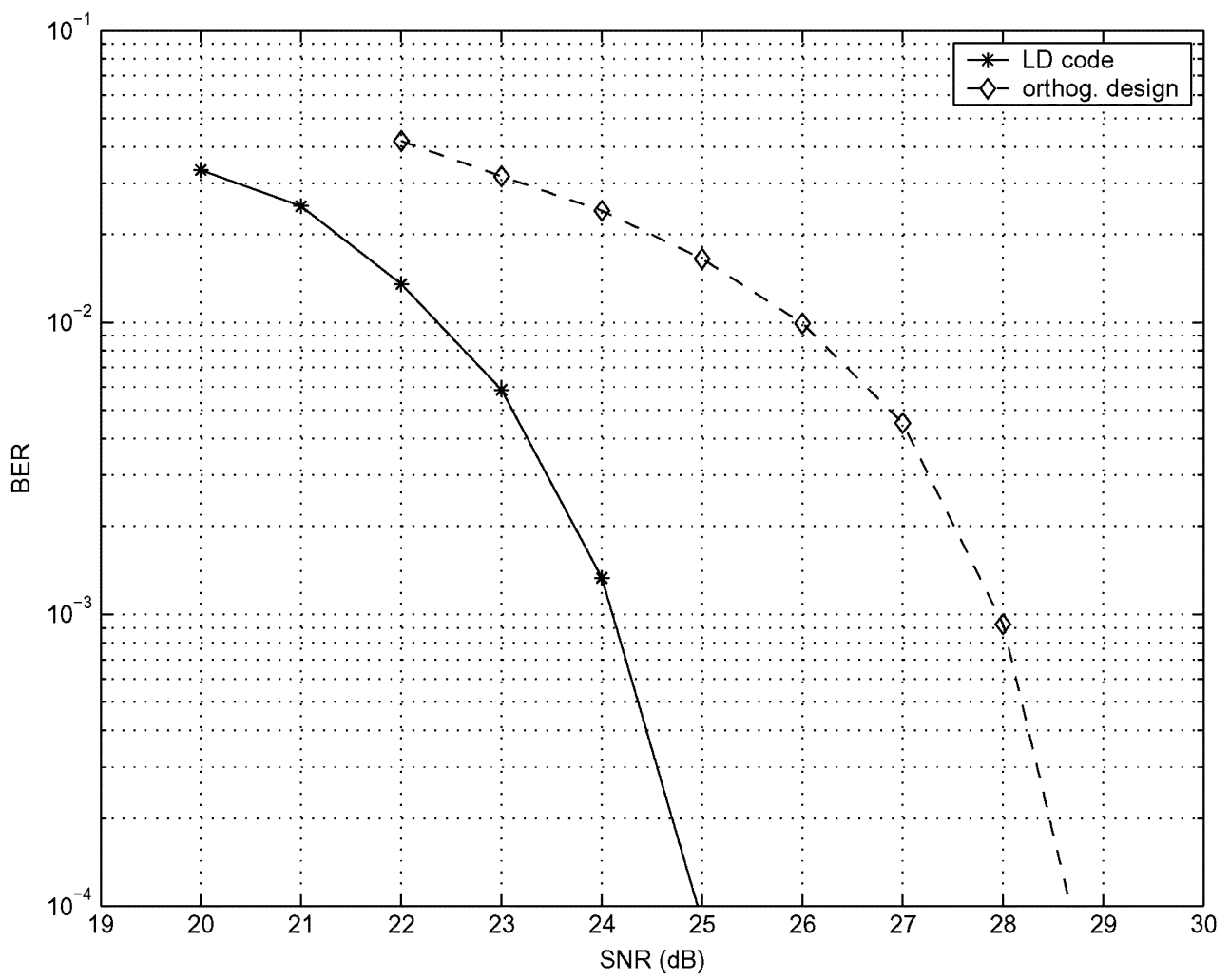

Fig. 11. Comparison of BER performances of LD code versus orthogonal design on $M=3, N=1$ system. Both schemes employ rate 8/9 LDPC code. The system using the LD code uses 64-QAM; the system using orthogonal design uses 256-QAM so that both have rate $R=6$. The FP-MAP is used for detection.

We have focused on LD codes. One can ask whether any S-T code would suffice, i.e., would any modulation scheme support the excellent performance of the powerful channel codes? Based on extensive simulations, we believe that the answer is negative: S-T modulation schemes need to maximize the mutual information between the input and output signals. In fact, the dispersion 
matrices of the LD code are actually chosen to maximize the mutual information between $x$ and $s$

$$
\begin{aligned}
& C_{\mathrm{LD}}(\rho, T, M, N) \\
& \quad=\max _{A_{q}, B_{q}, q=1, \ldots, Q} \frac{1}{2 T} E \log \operatorname{det}\left(I_{2 N T}+\frac{\rho}{M} \mathcal{H} \mathcal{H}^{\prime}\right)
\end{aligned}
$$

for a particular $\rho$ of interest and an appropriate power constraint. So, the LD codes satisfy the required criterion. Interestingly, V-BLAST also satisfies this condition. Indeed, as shown in Fig. 10, the multiantenna system employing an LDPC channel code and V-BLAST performs almost as well as the scheme with an LD code ([19, (36)]). Of course, there is an additional gain that the LD code obtains by spreading the signals across space and time more efficiently than V-BLAST. On the other hand, if an S-T code throws away some information [i.e., if it violates (20)], then the system performance is inferior in comparison to the $\mathrm{S}-\mathrm{T}$ code designed with (20) in mind. This is illustrated in Fig. 11, where we compare performance of the LD code of Fig. 9 with the orthogonal S-T code ([3]; also $[19,(35)])$. The orthogonal design does not satisfy (20) and the performance of the system is clearly much worse than of that employing the LD code.

\section{CONCLUSION}

In this paper, we developed a modification of the sphere decoding algorithm to perform the MAP detection and efficiently estimate soft information. We considered the expected complexity of the algorithm and found it is closely related to the expected complexity of the original sphere decoding. In fact, over a wide range of rates and SNR, the FP-MAP algorithm has expected complexity that is polynomial in the number of transmit antennas.

When combined with soft iterative decoding schemes, the proposed detection algorithm FP-MAP provides close to capacity performances of multiantenna systems. This was demonstrated on systems employing both turbo and LDPC codes. Furthermore, we illustrated performance of the FP-MAP algorithm in multiantenna systems that employ linear dispersive space-time modulation scheme as well as powerful LDPC channel codes. Our results imply that in order to obtain the remarkable performance of the iterative decoding, the space-time techniques need to optimize for the mutual information between the transmitted and received symbols. Thus, the design paradigms that constructs codes based on mutual information appear to be very reasonable.

Detailed quantitative analysis of the FP-MAP algorithm, its comparison with other decoding techniques, and determining regimes where a particular technique is superior to the others is certainly worth examining and may be investigated in future work.

\section{REFERENCES}

[1] I. E. Telatar, "Capacity of multi-antenna Gaussian channels," Eur. Trans. Telecom., vol. 10, pp. 585-595, Nov. 1999.
[2] V. Tarokh V, N. Seshadri, and A. R. Calderbank, "Space-time codes for high data rate wireless communication: Performance criterion and code construction," IEEE Trans. Inform. Theory, vol. 44, pp. 744-765, Mar. 1998.

[3] B. M. Hochwald and T. L. Marzetta, "Unitary space-time modulation for multiple-antenna communication in Rayleigh flat-fading," IEEE Trans. Inform. Theory, vol. 46, pp. 543-564, Mar. 2000.

[4] G. J. Foschini, "Layered space-time architecture for wireless communication in a fading environment when using multi-element antennas," Bell Labs. Tech. J., vol. 1, no. 2, pp. 41-59, 1996.

[5] E. Viterbo and J. Boutros, "A universal lattice code decoder for fading channels," IEEE Trans. Inform. Theory, vol. 45, pp. 1639-1642, July 1997.

[6] U. Fincke and M. Pohst, "Improved methods for calculating vectors of short length in a lattice, including a complexity analysis," Math. Comput., vol. 44, no. 4, pp. 463-471, Apr. 1985.

[7] M. O. Damen, A. Chkeif, and J. C. Belfiore, "Lattice code decoder for space-time codes," IEEE Commun. Lett., vol. 4, pp. 161-163, May 2000.

[8] B. Hassibi and H. Vikalo, "Expected complexity of the sphere decoder algorithm,", submitted for publication.

[9] C. Berrou, A. Glavieux, and P. Thitimajshima, "Near Shannon limit error-correcting coding and decoding. Turbo codes," in Proc. Int. Conf. Communications, 1993, pp. 1064-1070.

[10] J. P. Woodard and L. Hanzo, "Comparative study of turbo decoding techniques: An overview," IEEE Trans. Veh. Technol., vol. 49, pp. 2208-2233, Nov. 2000.

[11] R. G. Gallager, Low-Density Parity-Check Codes. Cambridge, MA: MIT Press, 1963.

[12] D. J. C. MacKay and R. M. Neal, "Near Shannon limit performance of low-density parity-check codes," Electron. Lett., vol. 32, pp. 1645-1646, 1996.

[13] D. J. C. MacKay, "Good error correcting codes based on very sparse matrices," IEEE Trans. Inform. Theory, vol. 45, pp. 399-431, Mar. 1999.

[14] A. Stefanov and T. M. Duman, "Turbo-coded modulation for systems with transmit and receive antenna diversity over block fading channels: System model, decoding approaches, and practical considerations," IEEE J. Select. Areas Commun., vol. 19, pp. 958-968, May 2001.

[15] P. D. Alexander, A. J. Grant, and M. C. Reed, "Iterative detection in code-division multiple-access with error control coding," Eur. Trans. Tel., vol. 9, pp. 419-425, 1998.

[16] H. Vikalo and B. Hassibi, "Low-complexity iterative decoding over multiple antenna channels via a modified sphere decoder," in Proc. Allerton Conf. Commun., Control, and Computing, Monticello, IL, Oct. 2001.

[17] B. M. Hochwald and S. ten Brink, "Achieving near-capacity on a multiple-antenna channel," in Proc. Allerton Conf. Commun., Control, and Computing, Monticello, IL, Oct. 2001.

[18] L. Bahl, J. Cocke, F. Jelinek, and J. Raviv, "Optimal decoding of linear codes for minimizing symbol error rate," IEEE Trans. Inform. Theory, pp. 284-287, Mar. 1974.

[19] B. Hassibi and B. Hochwald, "High-rate codes that are linear in space and time," IEEE Trans. Inform. Theory, vol. 48, no. 7, pp. 1804-1824, July 2002.

[20] P. Robertson, P. Hoeher, and E. Villebrun, "Optimal and suboptimal maximum a posteriori algorithms suitable for turbo decoding," Euro. Trans. Telecommun., vol. 8, pp. 119-125, Mar.-Apr. 1997.

[21] M. Tuechler, A. Singer, and R. Koetter, "Minimum mean squared error equalization using a-priori information," IEEE Trans. Signal Processing, vol. 50, pp. 673-683, 2002.

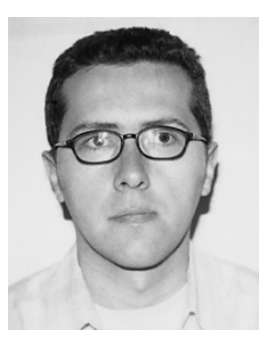

H. Vikalo was born in Tuzla, Bosnia and Herzegovina. He received the B.S. degree from the University of Zagreb, Croatia, in 1995, the M.S. degree from Lehigh University, Bethlehem, PA, in 1997, and the Ph.D. degree from Stanford University, Stanford, CA, in 2003, all in electrical engineering.

He held a short-term appointment at Bell Laboratories, Murray Hill, NJ, in the summer of 1999. From November 2002 to July 2003, he was a Postdoctoral Researcher, and since July 2003, he has been an Associate Scientist at the California Institute of Technology, Pasadena. His research interests include wireless communications, signal processing, estimation, and genomic information processing. 


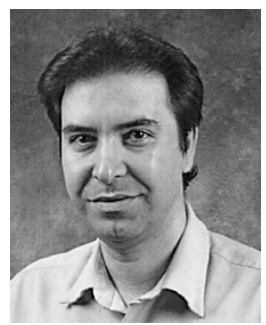

B. Hassibi was born in Tehran, Iran, in 1967. He received the B.S. degree from the University of Tehran in 1989 and the M.S. and Ph.D. degrees from Stanford University, Stanford, CA, in 1993 and 1996, respectively, all in electrical engineering.

From 1996 to 1998 he was a Research Associate with the Information Systems Laboratory, Stanford University. From 1998 to 2000 he was a Member of Technical Staff in the Mathematical Sciences Research Center, Bell Laboratories, Murray Hill, NJ. Since January 2001 he has been an Assistant Professor of electrical engineering at the California Institute of Technology, Pasadena. He has also held short-term appointments at Ricoh California Research Center, the Indian Institute of Science, and Linkoping University, Sweden. His research interests include wireless communications, robust estimation and control, adaptive signal processing, and linear algebra. He is the coauthor of Indefinite Quadratic Estimation and Control: A Unified Approach to $\mathrm{H}^{2}$ and $\mathrm{H}^{\infty}$ Theories (New York: SIAM, 1999) and Linear Estimation (Englewood Cliffs, NJ: Prentice-Hall, 2000).

Prof. Hassibi received the 1999 O. Hugo Schuck Best Paper Award of the American Automatic Control Council, a 2002 National Science Foundation Career Award, and a 2003 Okawa Foundation Research Grant for Information and Telecommunications.

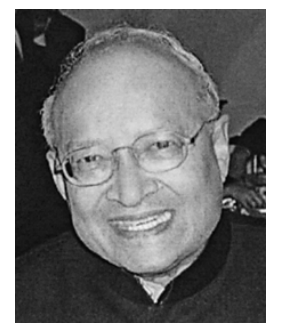

T. Kailath received the B.E. (Telecom) degree from Pune University, India, and the S.M. and Sc.D. degrees in electrical engineering from the Massachusetts Institute of Technology, Cambridge.

He has also been awarded honorary degrees from Linkoping University, Sweden, Strathclyde University, Scotland, the University of Carlos III, Madrid, Spain, and the University of Bordeaux, France. From 1961 to 1962 , he was with the Jet Propulsion Laboratories, Pasadena, CA, and also taught part-time at the California Institute of Technology. Since then he has been with Stanford University, where he is currently Hitachi America Professor of Engineering, Emeritus. He has also held shorter term appointments at several institutions around the world. His research has spanned a large number of disciplines, emphasizing information theory and communications in the 1960s, linear systems, estimation and control in the 1970s, VLSI design and sensor array signal processing in the 1980 s, and applications to semiconductor manufacturing and digital communications in the 1990s. Concurrently, he contributed to several fields of mathematics, especially stochastic processes, operator theory, and linear algebra. In the course of his research and teaching, he has mentored more than a hundred doctoral and postdoctoral students and authored or coauthored more than 300 journal papers. His books include Linear Systems (Englewood Cliffs, NJ: Prentice-Hall, 1980), Indefinite Quadratic Estimation and Control (with B. Hassibi and A. H. Sayed) (SIAM, 1999), and Linear Estimation (with A. H. Sayed and B.Hassibi) (Englewood Cliffs, NJ: Prentice-Hall, 2000). He has held Guggenheim, Churchill, and Humboldt fellowships, among others.

Prof. Kailath is a member of the National Academy of Engineering and the National Academy of Sciences,the American Academy of Arts and Sciences, the Third World Academy of Sciences, the Indian National Academy of Engineering, and the Royal Spanish Academy of Engineering. He has received outstanding paper prizes from the IEEE Information Theory Society, the IEEE Signal Processing Society, the European Signal Processing Society, and the IEEE TRAnsactions on SEMiconductor Manufacturing. He served as President of the IEEE Information Theory Society in 1975, and received its Shannon Award in 2000. Among other awards are the Technical Achievement (1989) and Society (1991) Awards of the IEEE Signal Processing Society, the 1995 IEEE Education Medal, and (with A. Sayed) the 1996 IEEE Donald G. Fink Prize Award. 